\section{\$27. On Flow Reversal in Turbulent Swirling Flow}

Itho, $\mathrm{K}$.

Itoh, S.-I. (RIAM, Kyushu Univ.)

Yokoi, N., Yoshizawa, A. (Univ. Tokyo)

In this work, turbulent swirling flow is studied with the aid of a variational method. The mean-flow field is expressed by the mean vorticity proportional to its curl. The total axial flux and total helicity per unit length are introduced to characterize the swirling flow, and the condition for the reversal of the axial flow near the center of flow is obtained. On this basis, the helicity injection is discussed in the light of the control of the onset of flow reversal in a turbulent swirling flow. Reference is also made to a cylindrical jet in relation to a swirling laboratory jet and an accretion-disk jet.[1]

A variational principle has been constructed for the fully-developed fluid turbulence, such that the turbulent dissipation

$\Phi$ takes minimum,

while the mean flow $\Psi$ is conserved [2], where

$$
\Phi=\int_{V}\left(\frac{\partial U_{j}}{\partial x_{i}}\right)^{2} \mathrm{~d} V, \boldsymbol{\Psi}=\int_{V} \boldsymbol{U} \cdot \boldsymbol{W} \mathrm{d} V
$$

By this variational principle, an explanation for the flow reversal phenomena was given [2], and the comparison with experimental observation has been in progress [3].

We obtain a condition for the flow reversal as

$$
|H|>H_{c} \equiv \frac{K}{\pi^{2} x_{1}^{(1)}} \frac{F^{2}}{r_{M}^{3}}
$$

with $H / U_{\theta}<0$. When the total helicity per unit length, $H$, exceeds the threshold $H_{c}$ that is determined by the total flux $F$ and the system size $r_{M}$, the reversal of axial flow takes place. (Here, $K=2 \pi x^{(1)^{2}} J_{0}^{2}\left(x^{(1)}\right)$ is a geometrical coefficient.) In figure 1, a diagram is shown on the plane of $(H, F)$ for the reversal, retardation and acceleration of axial flow velocity at the axis.

First, this result is applied to the astrophysical jet, in which the axial flux of angular momentum flux plays the key role in the rate of accretion. In this system, the helicity and the axial flux of angular momentum $P$ has a relation as

$$
|H|=\frac{\pi x_{(1)^{3}}}{2 r_{M}} \frac{p^{2}}{F^{2}}
$$

We obtain the critical limit of $P$ as

$$
P>P_{c} \equiv-\frac{2 J_{0}\left(x_{1}^{(1)}\right)}{\pi x_{1}^{(1)}} \frac{F^{2}}{r_{M}}
$$

This limits the rate of the loss of angular momentum in the astrophysical jet. That is, the rate of the release of gravitational energy in the accretion star is limited by this, if the axial jet is the primary mechanism of the angular momentum.

Next, we study swirling jets which are familiar in engineering flows. In the case that a jet is ejected into the surrounding fluid at rest, the mean velocity profile, specifically, the axial velocity, differs considerably at the outer side of the jet, compared with the foregoing cylindrical jet. The formalism developed in this article is based on the global parameters such as the mass and angular momentum fluxes, not so sensitive to the details of flow structures. Then it is meaningful to compare the present analytic criteria with observations of laboratory jets.

In a study of swirling jets, the swirl number $S=P / M R_{S}$ is a characteristic nondimensional parameter. Here $M$ is the axial thrust (per unit mass) and $R_{s}$ is the swirl generator radius. We have the critical condition for reversal as

$$
S>S_{c}=\frac{-2 J_{0}\left(x^{(1)}\right)}{|\lambda| R_{S}\left(1+J_{0}^{2(}\left(x_{1}^{(1)}\right) / 2\right)},
$$

which results in $S_{c}=0.4$. In observations of swirling jets, the critical swirl number for the flow reversal is reported as $S_{c} \simeq 0.64$.

It is stressed that the sign of $H / U_{\theta}$ (the ratio of the total helicity to the characteristic swirl velocity) is the key for the induction of the retardation/reversal of the axial flow. The condition of the axial flow reversal is derived, which indicates the importance of the helicity injection when the flow is accelerated by swirling.

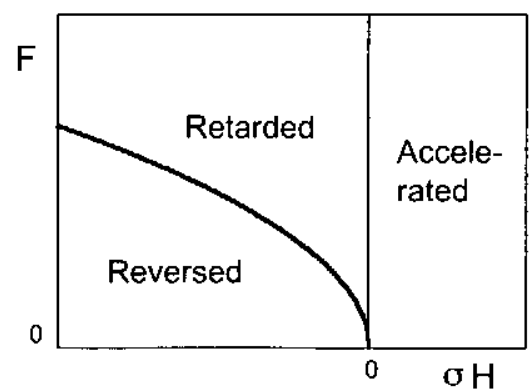

Fig. 1. Domain of reversal, retardation and acceleration at the central flow velocity is illustrated.

\section{References}

[1] K. Itoh, S.-I. Itoh, N. Yokoi, A. Yoshizawa: J. Phys. Soc. Jpn. 72 (2003) 2781

[2] A. Yoshizawa, N. Yokoi, S. Nisizima, S.-I. Itoh and K. Itoh: Phys. Fluids 13 (2001) 2309.

[3] N. Yokoi, et al. (2004) paper in print. 